Discriminating the trapped electron modes contribution in density fluctuation spectra

This content has been downloaded from IOPscience. Please scroll down to see the full text. 2015 Nucl. Fusion 55093021

(http://iopscience.iop.org/0029-5515/55/9/093021)

View the table of contents for this issue, or go to the journal homepage for more

Download details:

IP Address: 157.193.64.157

This content was downloaded on 01/12/2015 at $11: 10$

Please note that terms and conditions apply. 


\title{
Discriminating the trapped electron modes contribution in density fluctuation spectra
}

\author{
H. Arnichand ${ }^{1}$, R. Sabot ${ }^{1}$, S. Hacquin ${ }^{1}$, A. Krämer-Flecken ${ }^{2}$, C. Bourdelle ${ }^{1}$, \\ J. Citrin ${ }^{1,3}$, X. Garbet ${ }^{1}$, J.C. Giacalone ${ }^{1}$, R. Guirlet ${ }^{1}$, J.C. Hillesheim ${ }^{4}$, \\ L. Meneses ${ }^{5}$ and JET Contributors ${ }^{\mathrm{a}}$ \\ EUROfusion Consortium, JET, Culham Science Centre, Abingdon, OX14 3DB, UK \\ ${ }^{1}$ CEA, IRFM, F-13108 Saint-Paul-Lez-Durance, France \\ 2 Institute for Energy Research (Plasma Physics) Forschungszentrum Jülich, D-52425 Jülich, Germany \\ ${ }^{3}$ FOM Institute DIFFER-Dutch Institute for Fundamental Energy Research, Nieuwegein, \\ The Netherlands \\ ${ }^{4}$ Culham Centre for Fusion Energy, Culham Science Centre, Abingdon, OX14 3DB, UK \\ 5 Instituto de Plasmas e Fusão Nuclear, Instituto Superior Técnico, Universidade Técnica de Lisboa, \\ 1049-001 Lisboa, Portugal
}

E-mail: hugo.arnichand@cea.fr

Received 15 January 2015, revised 1 July 2015

Accepted for publication 9 July 2015

Published 17 August 2015

\begin{abstract}
Quasi-coherent (QC) modes have been reported for more than 10 years in reflectometry fluctuations spectra in the core region of fusion plasmas. They have characteristics in-between coherent and broadband fluctuations as they oscillate at a marked frequency but have a wide spectrum. This work presents further evidences of the link recently established between QC modes and the trapped electron modes (TEM) instabilities (Arnichand et al 2014 Nucl. Fusion 54 123017). In electron cyclotron resonance heated discharges of Tore Supra, an enhancement of QC modes amplitude is observed in a region where TEM cause impurity transport and turbulence. In JET Ohmic plasmas, QC modes disappear during density ramp-up and current ramp-down. This is reminiscent of Tore Supra and TEXTOR observations during transitions from the linear Ohmic confinement (LOC) to the saturated Ohmic confinement (SOC) regimes. Evidencing TEM activity then becomes experimentally possible via analysis of fluctuation spectra.
\end{abstract}

Keywords: turbulence, micro-instabilities, trapped electron modes, electron cyclotron resonance heating, ohmic confinement, reflectometry

(Some figures may appear in colour only in the online journal)

\section{Introduction}

In magnetic fusion devices, turbulent transport is often attributed to long wavelength instabilities whose perpendicular wave-number $k_{\perp}$ normalized to the ion Larmor radius $\rho_{i}$ is between $0.1<k_{\perp} \rho_{i}<1$. At this scale, two micro-instabilities are frequently dominant in core tokamak plasmas: ion temperature gradient (ITG) and trapped electron modes (TEM) [1]. As different instabilities usually coexist, several modes

\footnotetext{
${ }^{a}$ See the appendix of Romanelli et al (2014 Proc. of the 25th IAEA Fusion Energy Conf. (Saint Petersburg, Russia))
}

can couple to each other to give a hybrid or mixed mode turbulence [2]. Discriminating ITG or TEM dominated regimes is then not always possible, and in any case challenging due to their similar scale. However, this is essential to investigate their effects on the plasma parameters and to validate turbulence models.

Although they can induce particle and heat transport of the similar magnitude, their contributions to the ratio $q_{i} / q_{e}$ (ion to electron heat flux ratio) or to the direction of the turbulent driven particle pinch, can be noticeably different. Discriminating ITG/TEM from particle or impurity transport requires an accurate evaluation of the diffusion coefficient and 
Table 1. Main characteristics of the reflectometers used.

\begin{tabular}{llll}
\hline Reflectometer properties & JET & TEXTOR & Tore Supra \\
\hline Wave polarization & X-mode & O-mode & X-mode \\
Location of the reflectometer & Low field side midplane & $\begin{array}{l}\text { Low field side midplane } \\
\text { and top of the torus }\end{array}$ & Low field side midplane \\
Probing frequency & $75-110 \mathrm{GHz}$ & $26-40 \mathrm{GHz}$ & $100-155 \mathrm{GHz}$ \\
Acquisition frequency & $2 \mathrm{MHz}$ & $2 \mathrm{MHz}$ & $1 \mathrm{MHz}$ \\
\hline
\end{tabular}
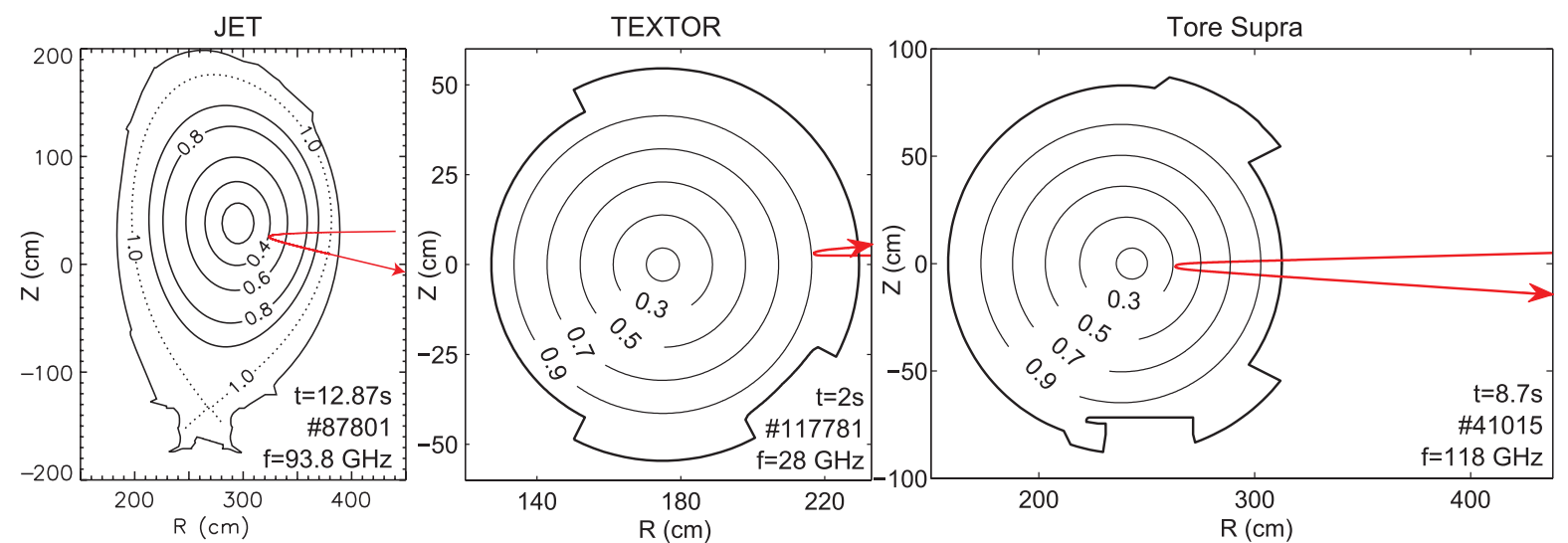

Figure 1. Example of ray tracing for JET, TEXTOR and Tore Supra reflectometers. The central rays emitted by the reflectometers are shown for data used in figure 7 . The divergence angle at the $-3 \mathrm{~dB}$ for the JET, TEXTOR and Tore Supra reflectometers are $7^{\circ}, 14^{\circ}$ and $2^{\circ}$ respectively.

the convection velocity. Ideal ITG and TEM rotate in opposite direction in the plasma frame, but the rotation velocity of the plasma itself is usually one order of magnitude higher [3]. Alternately, computation of the mode growth rates, based on measured plasma parameters is widely used to determine which mode is dominant [4]. However it has to be crosschecked with other methods because it relies on theoretical predictions and the fastest growing linear mode is not necessarily the one which dominates the turbulent transport.

The identification of direct spectral signatures of fluctuations induced by each instability would be a noticeable progress in turbulence investigations. A study combining Tore Supra and TEXTOR data analysis has recently suggested that this might be possible [5]. Indeed, spectra analysis has evidenced that TEM are linked with quasi-coherent (QC) modes observed by reflectometry in the core/edge region [6-8]. Besides the link established between QC modes and TEM, spectra modifications have also been reported during transitions toward expected TEM-dominated regimes in DIII-D [9-13], and Alcator C-mod [13-17] tokamaks, and in the MST reversed field pinch [18].

Fluctuation spectra showing features similar to QC modes are also observed at the very edge of the plasma in several advanced regimes (H-modes [19-22], Enhanced $D_{\alpha} \mathrm{H}$-modes [23-27], I-mode [28-30]). There is no unified explanation for these modes which present similar QC spectral signature. To interpret them, several instabilities have been proposed. None of these studies have suggested that TEM could be the underlying mechanism of the various QC oscillations observed at the very edge. Indeed, TEM are not expected to be unstable in this highly collisional region. To avoid confusion with them, we choose to name QC-TEM the modes linked with TEM that we investigate in this paper.
In this article we present a detailed analysis of reflectometry fluctuation spectra in electron cyclotron resonance heating $(\mathrm{ECRH})$ and Ohmic plasmas, both supporting the link between QC modes and TEM previously evidenced [5]. Section 2 presents the diagnostics set-up and section 3 describes the main properties of QC-TEM. In section 4, we show the ECRH effects on QC-TEM in a Tore Supra region where Nickel transport analysis and linear growth rate computation gives indications on the dominant instability. Section 5 presents reflectometry spectra in density ramp-up and current ramp-down performed in JET Ohmic plasmas. A qualitative agreement is found with recent Tore Supra and TEXTOR observations.

\section{Diagnostics set-up}

This analysis uses conventional reflectometers to study density fluctuation in TEXTOR [31], Tore Supra [32] and JET $[33,34]$. Their main properties are summarized in table 1 and an example of ray tracing is shown for each reflectometer in figure 1. Conventional reflectometry consists in probing the plasma with an electromagnetic wave, which is reflected at the so-called cut-off layer, and received by the reflectometer. To study density fluctuations at a given position, the probing frequency is kept constant during the time range of interest for spectral analysis. Nonetheless, to probe the plasma at different radial positions, several frequency steps are usually done within a discharge.

I-Q heterodyne systems now commonly used by reflectometers provide $A(t) \cos (\phi(t))$ and $A(t) \sin (\phi(t))$, where $A(t)$ and $\phi(t)$ are the amplitude and the phase, respectively. Hence, the 


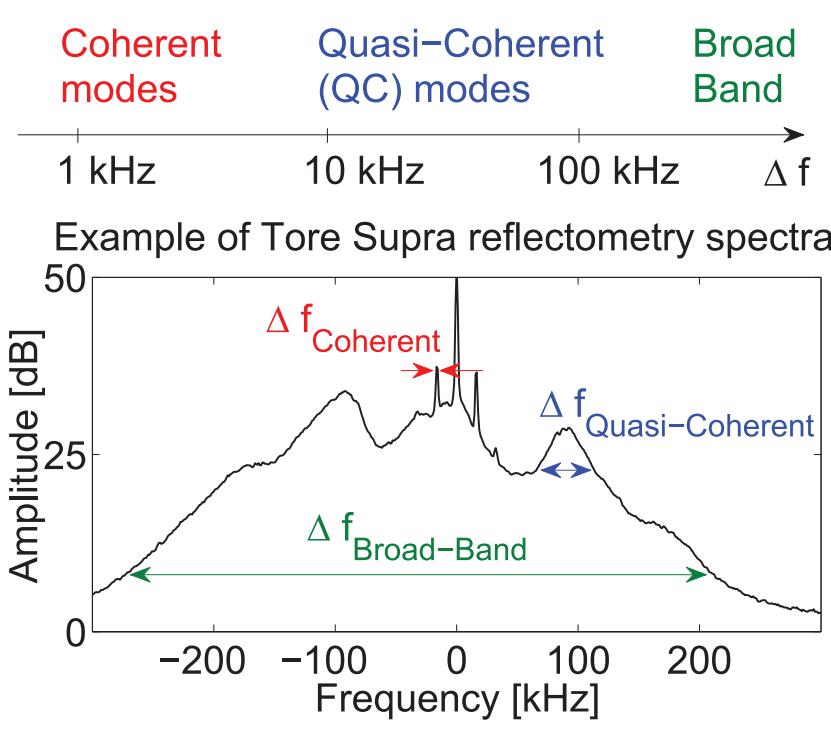

Figure 2. Example of reflectometry spectra from Tore Supra (\# 40805) with its different types of component as a function of their frequency width: coherent (in red), quasi-coherent (in blue) and broad-band (in green).

reflectometer signal can be seen as a complex signal $A(t) \mathrm{e}^{\mathrm{i} \phi(t)}$. The Fourier transform of this complex signal provides spectra with positive and negative frequencies, corresponding to the phase increments and decrements respectively.

Contrary to spectra of Doppler backscattered signals [35], conventional reflectometry spectra are expected to be rather symmetric. However, a small asymmetry is always observed between positive and negative frequencies. It can arise from the combination of different phenomena (Doppler shift induced by plasma rotation, misalignment of the antenna, vertical shift of the plasma position, sawteeth, etc) [36].

\section{Review of QC-TEM properties}

\subsection{Spectral signature of QC-TEM}

As previously introduced, in T-10 [7], TEXTOR [6] and Tore Supra [5], reflectometry fluctuation spectra can show QC oscillations $(30<f<150 \mathrm{kHz})$ in different regimes. As shown in figure 2 , QC modes $(\Delta f \approx f)$ are in-between narrow peaks associated to coherent modes such as MHD or GAM $(\Delta f \leqslant f)$ and broad-band fluctuations induced by turbulence $(\Delta f \gg f)$. They were termed quasi-coherent because they oscillate around a given frequency but have an intermediate status, reminiscent of broad-band fluctuations.

As discussed in the previous section, an asymmetry between positive and negative frequencies may be observed in spectra from 'conventional reflectometry'. An additional remark on this asymmetry is necessary to define properly a QC oscillation which has to be distinguished from a spectra peak induced by Doppler effect. In the case of Doppler shift, a peak is seen in one side of the spectra only, therefore the spectra is not centered at the zero frequency [35]. On the contrary, QC modes induce two peaks, both in the positive and the negative frequencies.
3.2. Perpendicular velocity and normalized scale of QC-TEM

In TEXTOR, the multiple antenna system have the special feature to perform poloidal and toroidal correlation reflectometry. Indeed, the several antenna arrays installed at different poloidal and toroidal locations [31] allow to perform shortrange poloidal correlation and long-range toroidal correlation.

Long-range toroidal correlation shows that QC-TEM modes are the only oscillations which clearly appear in TEXTOR coherence spectra [37]. This has recently allowed to evidence their burst like character and their 3-dimensional nature [8].

Short-range poloidal correlation allows to estimate the perpendicular rotation. These measurements provide the total perpendicular velocity:

$$
\Omega_{\perp}^{\text {measured }}=\Omega_{\perp}^{E \times B}+\Omega_{\perp}^{\text {phase }}
$$

With $\Omega_{\perp}^{E \times B}$ the rotation due to the mean $E \times B$ drift, and $\Omega_{\perp}^{\text {phase }}$ the rotation due to the average phase velocity of the mode in the plasma frame. Such measurements were performed from the LFS and the top of TEXTOR. They have shown from the top of the torus only, that QC-TEM rotate faster in the electron diamagnetic direction than lower broadband frequency fluctuations [5]. As $\Omega_{\perp}^{E \times B}$ is constant at a given position and time, only a distinct $\Omega_{\perp}^{\text {phase }}$ can explain such velocity difference $\left(400 \mathrm{~m} \mathrm{~s}^{-1}\right)$. It suggests that $\Omega_{\perp}^{\text {phase }}$ is oriented in the electron diamagnetic direction for the QC-TEM (as expected for electron-driven modes) and/or in the ion diamagnetic direction for the low frequencies (as expected for ion-driven modes).

Short-range correlation has also provided an estimation of the poloidal wavelength associated with these QC-TEM oscillations $\left(0.5<k_{\perp}<3\left[\mathrm{~cm}^{-1}\right]\right)$ and their poloidal mode number $(20<m<70)$ [6]. An estimation of their normalized scale $k_{\perp} \rho_{i}$ is also possible. Due to the lack of $T_{i}$ measurements, a typical ion temperature profile has to be used $\left(T_{i}(r / a)=T_{i}(0)\left[1-(r / a)^{2}\right]^{2}\right)$, with $T_{i}(0)[\mathrm{eV}]$ the central ion temperature provided by the Artsimovich's formula valid for Ohmic plasmas [38]:

$$
T_{i}(0)=(1.29 \pm 0.11) \frac{\left(I_{\mathrm{p}} \cdot B_{t} \cdot R^{2}<n>\right)^{1 / 3}}{\sqrt{A_{i}}}
$$

Where $I_{\mathrm{p}}[\mathrm{kA}]$ is the plasma current, $B_{t}[\mathrm{kG}]$ is the toroidal magnetic field, $R[\mathrm{~cm}]$ is the tokamak major radius, $n$ $\left[10^{13} \mathrm{~cm}^{-3}\right]$ is the line-averaged plasma density, and $A_{i}$ is the atomic mass of the working gas. Figure 3 shows the estimated normalized scale of a set of TEXTOR Ohmic discharges. It results that $0.4 \leqslant k_{\perp} \rho_{i} \leqslant 0.5$, in good agreement with previous observations always in the range $k_{\perp} \rho_{i} \leqslant 1$ [6-8]. Such normalized scale is in the range of micro-instabilities such as TEM and ITG.

\subsection{Radial and poloidal location of QC-TEM}

Combining the several reflectometers installed in different tokamaks, indications on the location of QC-TEM can be provided. Both poloidal and radial location of QC-TEM is in agreement with expectation for micro-instabilities: 


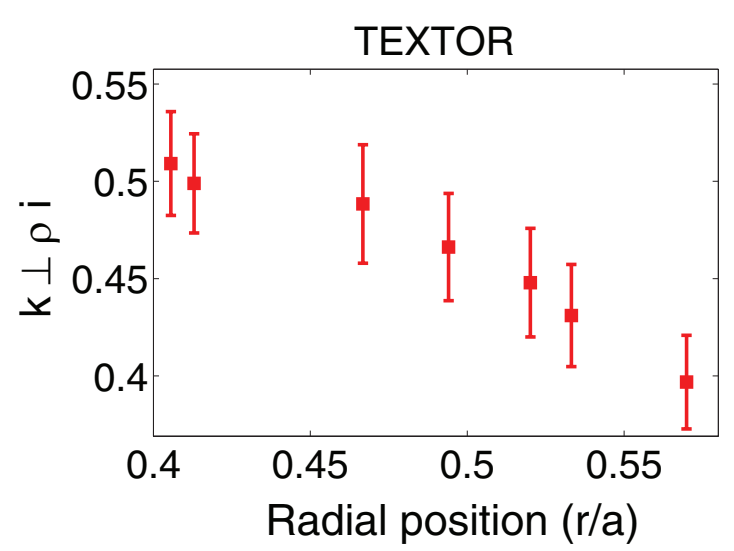

Figure 3. Normalized scale of QC-TEM estimated with equation (2) for different radial position at the LFS of TEXTOR Ohmic plasmas (\#113802-7) with $B_{t}=1.9 \mathrm{~T}, I_{\mathrm{p}}=400 \mathrm{kA}$ and $1.33<n_{e}\left[\mathrm{~m}^{-3} 10^{19}\right]<1.58$.
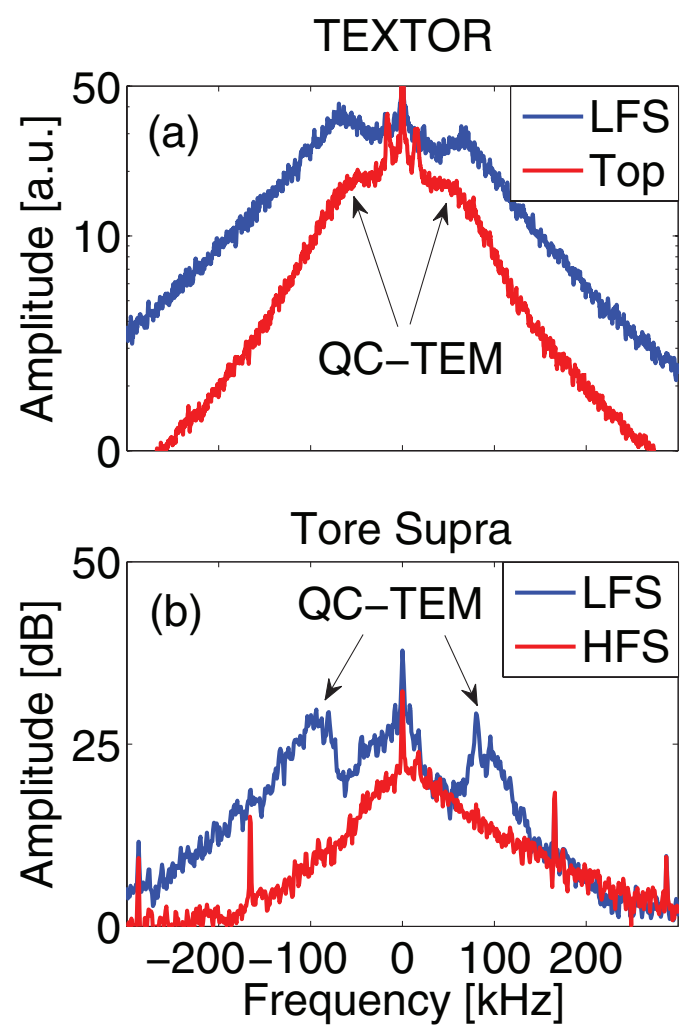

Figure 4. Poloidal ballooning of QC-TEM highlighted by reflectometry spectra from $(a)$ TEXTOR at $r / a \approx 0.84$ between the LFS (\#116492) and the top of the torus (\#116495) and (b) Tore Supra between the LFS and the HFS (\#40801 at $r / a \approx 0.11$ ).

- Radially, QC-TEM can appear at many different positions in the plasma core/edge region. They do not depend on a specific location such as the rational surfaces or the separatrix. As an example, observations of QC-TEM have been reported in Tore Supra from $r / a \approx 0.1$ until $r / a \approx 0.8$ [5], depending on the plasma scenario.

- Poloidally, TEXTOR and Tore Supra spectra show that the amplitude of QC-TEM is ballooned on the low field side (LFS) midplane. In TEXTOR, reflectometers can probe the plasma from the LFS midplane and from the

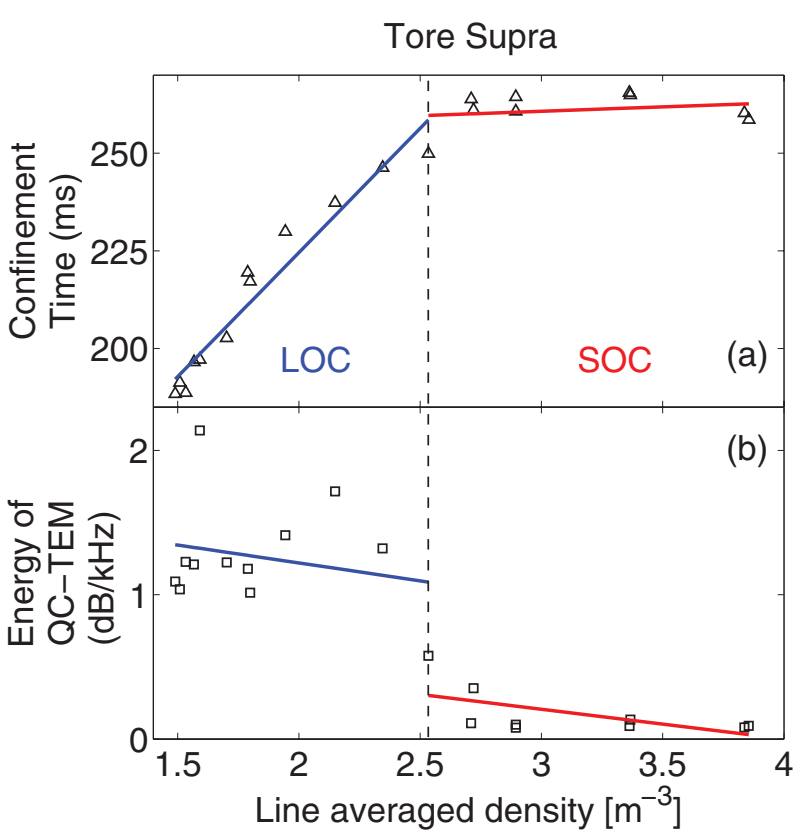

Figure 5. Confinement time (a) and energy of QC-TEM (b) as a function of the line averaged density for a set of Tore Supra discharges \#41003-41013 ( $\left.B_{t}=3.8 \mathrm{~T}, I_{\mathrm{p}}=1.2 \mathrm{MA}\right)$.

top of the torus. Usually, more pronounced QC-TEM are observed at the LFS than at the top (figure 4(a)). In Tore Supra, where the reflectometers are able to measure on the LFS and on the high field side (HFS), most of the time spectra can show QC-TEM on the LFS only (figure 4(b)).

These general properties of QC-TEM (mode number, normalized scale, radial and poloidal location, etc) pointed toward a link with micro-instabilities. Initial investigations suggested ITG as a driving mechanism $[6,7]$ but this interpretation has been re-evaluated since regimes in which these modes were observed contradict this interpretation [5].

\section{QC-TEM in Ohmic plasmas}

In Ohmic plasmas, the confinement time first increases linearly with density in the linear Ohmic confinement (LOC) regime and then saturates in the saturated Ohmic confinement (SOC) regime, as shown in figure 5(a). If the onset of an ion mode in the SOC regime was proposed to explain the energy confinement time saturation $[39,40]$, it is now widely believed that the electrons play a major role, as suggested in $[41,42]$. During the last decade, the conventional understanding of turbulence in Ohmic plasma is that the LOC regime is dominated by TEM and the SOC regime by ITG $[3,5,14,43-46]$.

The LOC-SOC transition is predicted to occur at $n_{\mathrm{s}}$ the so-called Shimomura density (in $10^{20} \mathrm{~m}^{-3}$ ) defined by: [47]:

$$
n_{\mathrm{s}}=\frac{I_{\mathrm{p}} \mu_{0}}{2 \pi a^{2}} \sqrt{\frac{A_{i} \kappa}{2}}
$$

where $a$ is the minor radius of the tokamak, $\kappa$ the plasma elongation, $A_{i}$ the plasma atomic mass number, $I_{\mathrm{p}}$ the plasma current and $\mu_{0}$ the vacuum permittivity. As shown by equation (3), 


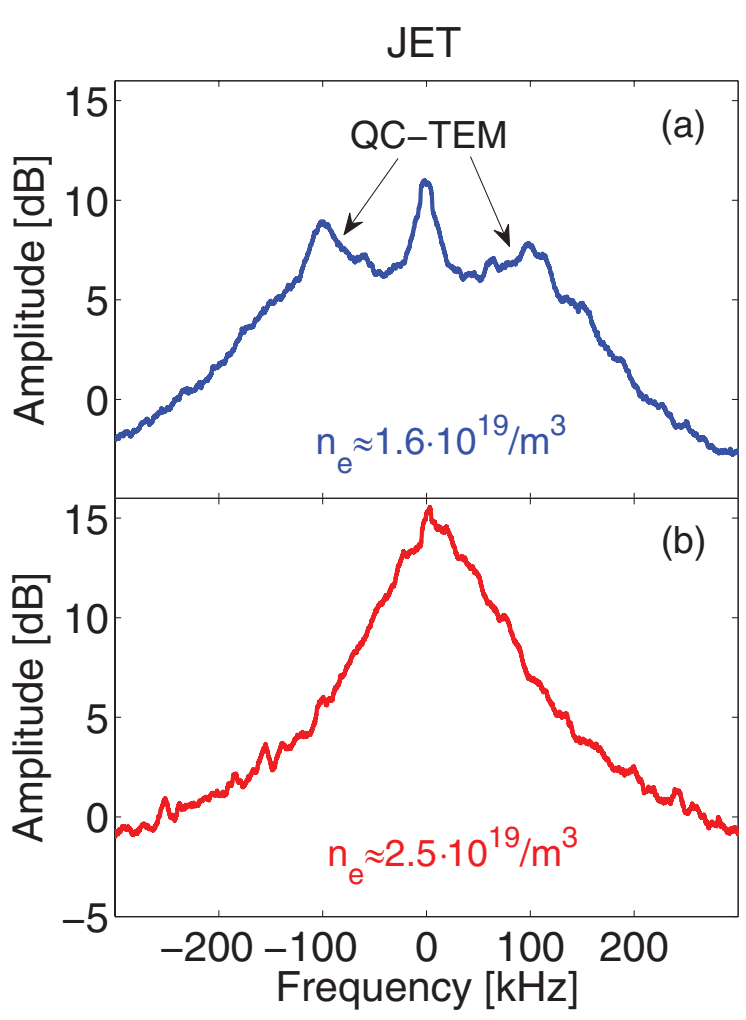

Figure 6. Fluctuation spectra from the JET reflectometer measured for $0.3<r / a<0.33$ during \#87756 $\left(B_{t}=1.8 \mathrm{~T}, I_{\mathrm{p}} \approx 1.8 \mathrm{MA}\right.$, limiter plasma). (a) shows measurements for $n_{e} \approx 1.6 \cdot 10^{19} \mathrm{~m}^{-3}$ with $f_{\text {reflecto }}=86.6 \mathrm{GHz}$ and $(b)$ for $n_{e} \approx 2.5 \cdot 10^{19} \mathrm{~m}^{-3}$ with $f_{\text {reflecto }}=76.4 \mathrm{GHz}$

$n_{\mathrm{s}}$ is proportional to the plasma current. Hence a transition from the LOC to the SOC regimes can also be achieved at constant density with a current ramp-down. In the following chapters, both density and current scans performed in Ohmic plasmas are analyzed. One can note that equation (3) provides the plasma parameters whose depends the transition density, but that it does not allow to recover the values of the transition experimentally observed. Hence it cannot be used to determine whether the plasma is in the $\mathrm{LOC}$ or the SOC regime.

\subsection{Stabilization of QC-TEM by density ramp-up in Tore Supra and JET}

The advantage of performing density ramp-up to investigate the LOC-SOC transition is that the transition can be rather well identified by looking at the confinement time which saturates in the SOC regime. For reflectometry measurements, the drawback of such analysis is the modification of the cut-off layer locations during the density scan $\left(r / a \propto n_{e}\right)$. Thus the probing frequency of the reflectometer has to be changed between each measurement to maintain a constant reflection layer in the plasma.

4.1.1. Tore Supra results. This was done to observe at $r / a \approx 0.3$ the evolution of QC-TEM in a set of Tore Supra discharges (\#41003-41013). Figure 5(b) shows that the 'energy' of QC-TEM decreases dramatically between the LOC regime $(\geqslant 1)$ and the SOC regime $(\leqslant 0.3)$. To obtain this energy, the power spectra have been decomposed in several Gaussians corresponding to each of its components (QC-TEM, broadband, etc). The energy of QC-TEM being defined by the ratio of the amplitude $[\mathrm{dB}]$ divided by the full width at half of its maximum $[\mathrm{kHz}]$ of the Gaussian fitting QC-TEM. Figure 5 shows that the QC-TEM stabilization and the LOC-SOC transition are correlated, which support the long-standing hypothesis predicting the stabilization of TEM in the SOC regime.

In these discharges charge exchange recombination spectroscopy were not acquired, thus measurements of $T_{i}$ and rotation are not available. However, two dedicated studies involving intrinsic rotation measurements and linear gyrokinetics simulations were performed in a similar Tore Supra experiment [5, 48].

From [5] it can be seen that the most unstable modes observed drifts in the electron diamagnetic direction in the LOC regime and in the ion diamagnetic direction in the SOC regime. This is consistent with the absence of QC-TEM when ITG is the dominant instability and their onset in TEMdominated regime. From [48] we can see that the toroidal rotation amplitude has been found to decrease as the electron density increased. No rotation reversal were observed as it was reported in several machines [15, 16, 44, 49, 50]. However, in the core $(r / a<0.5)$ a transitory acceleration is observed at the LOC-SOC transition. Such toroidal velocity bifurcation was correlated to a stabilization of QC-TEM observed inside this radial range [48] but also further out in the plasma $(0.6<r / a<0.7)[51]$ where no major change of intrinsic rotation is observed.

4.1.2. JET results. A density scan $\left(1.5<n_{e}<2.5\right)$ was also analyzed in JET $(R=2.96 \mathrm{~m}$ and $1.25<a[\mathrm{~m}]<2.10)$ during an Ohmic discharge in a limited configuration (\#87756). Figure 6 which presents reflectometry spectra at different densities shows that a similar picture than Tore Supra is found in JET: QC-TEM appears only at low density. This experiment was not dedicated to the present analysis, consequently the plasma parameters make hazardous the determination of the LOCSOC transition by confinement time estimation. Hence the comparison with Tore Supra observations remains qualitative.

\subsection{Stabilization of QC-TEM by current ramp-down in Tore Supra, JET and TEXTOR}

For reflectometry measurements, performing current scan has the advantage to keep rather constant the measurements location during the scan. The drawback of such analysis is to make more complicated the determination of the LOC-SOC transition which can then be determined by:

- In TEXTOR, the LOC-SOC transition has been determined by using previous study related to the Ohmic confinement regimes (see figure $7(b)$ ). In this machine, the transition is expected to occur at $n_{e} / n_{\mathrm{Gr}} \approx 0.55$ [52] with $n_{\mathrm{Gr}}$ the Greenwald density $\left(n_{\mathrm{Gr}}=I_{\mathrm{p}} / \pi a^{2}\right)$.

- In Tore Supra, the LOC and SOC regimes of figure 7(a) have been determined in another way: several density scans at different plasma current have been analyzed to 
(a) Tore Supra

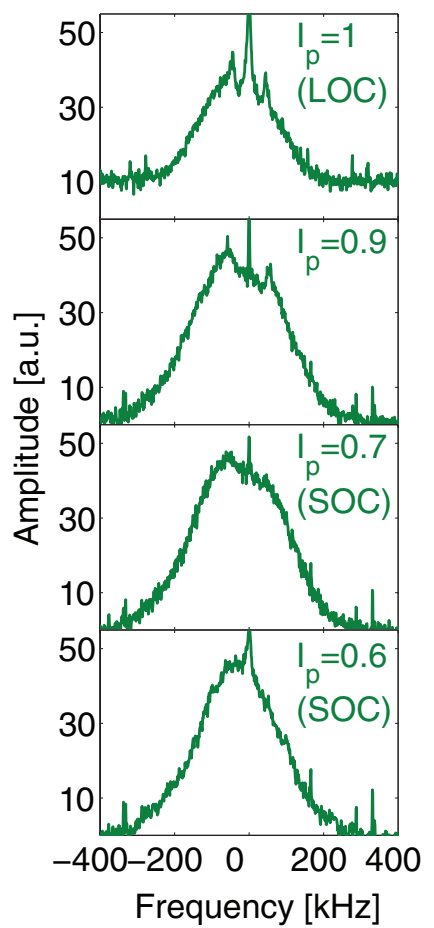

(b) TEXTOR

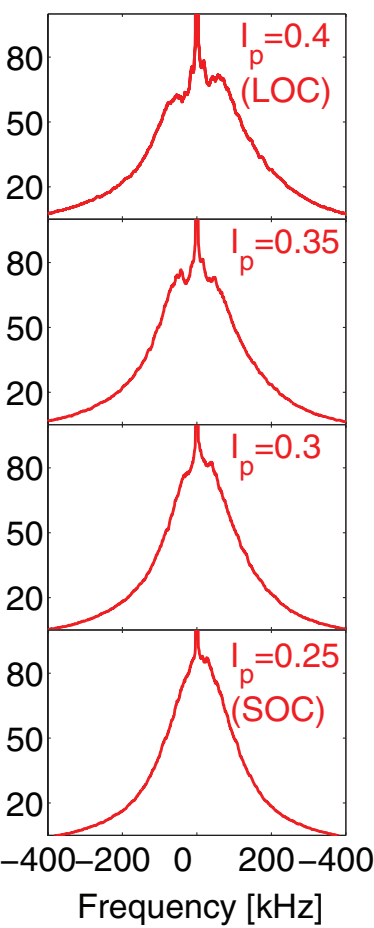

(c) JET

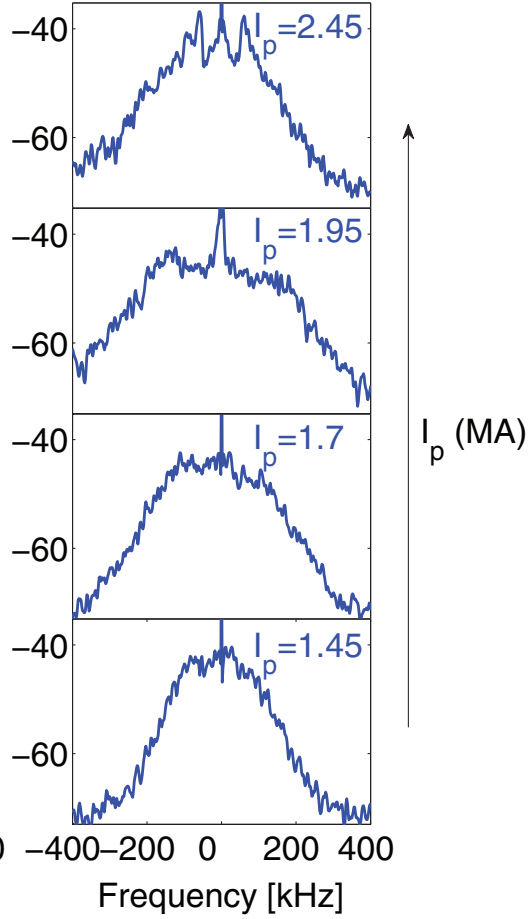

Figure 7. Reflectometry spectra in $\log$ scale from $(a)$ Tore Supra measured at $r / a \approx 0.3$ during a sampling time of $8 \mathrm{~ms}$ with $2.1<n_{e}\left[10^{19}\right.$ $\left.\mathrm{m}^{-3}\right]<2.5$ (\#40487 and \#41015-41018), (b) TEXTOR measured at $r / a \approx 0.91$ during a sampling time of $230 \mathrm{~ms}$ with $n_{e} \approx 3 \times 10^{19} \mathrm{~m}^{-3}$ (\#117781) and (c) JET measured at $0.36<r / a<0.46$ during a sampling time of $2 \mathrm{~ms}$ with $n_{e} \approx 1.65 \times 10^{19} \mathrm{~m}^{-3}(\# 87801,2,4,5)$.

plot the transition density as a function of the plasma current. Such plot which is shown in [16], can be used to determine at which plasma current the transition will occur for a given density.

- In JET, unfortunately none of the two methods described above can be used to identify the LOC and the SOC regimes during the current scan shown in figure $7(c)$. Hence the Ohmic regimes have not been determined and the comparison with the other devices remains qualitative.

Figure 7 shows that for both Tore Supra $(a)$ and TEXTOR $(b)$, QC-TEM observed at high current (LOC regime) disappear at low current (SOC regime). Figure 7(c) shows that a similar tendency has been found in JET where high plasma current $\left(I_{\mathrm{p}}=2.45\right.$ and $\left.1.95 \mathrm{MA}\right)$ show clear QC-TEM while lower plasma current shows no or weak QC-TEM $\left(I_{\mathrm{p}}=1.45 \mathrm{MA}\right)$.

\section{QC-TEM in Tore Supra ECRH plasmas}

ECRH has been widely used to destabilize TEM in core tokamak plasmas [9, 10, 43, 53]. Here, its effect on Nickel transport, linear gyro-kinetic simulations and reflectometry fluctuation spectra are investigated in Tore Supra $(R=2.4 \mathrm{~m}$, $a=0.72 \mathrm{~m}$, circular plasma).

\subsection{Impurity transport analysis: $R / L_{T_{e}}$ dependence of nickel diffusion}

A power of $250 \mathrm{~kW}$ of ECRH were deposited by two gyrotrons in Tore Supra discharges with $B_{t}=3.8 \mathrm{~T}$ and $I_{\mathrm{p}}=0.5$
MA. Keeping the total ECRH power constant, the deposition radii of the two gyrotrons were changed:

- Inner deposition at $r / a=0.35$ (\#40801)

- Outer deposition at $r / a=0.58$ (\#40807)

- Mixed deposition at $r / a=0.35$ and $r / a=0.58$ (\#40805).

Owing to this combination, we were able to modify the temperature gradient inside $r / a=0.3$ while keeping the density profile almost constant $[54,55]$. Switching ECRH on suppresses small sawteeth observed in the Ohmic phase $\left(T_{e}<100 \mathrm{eV}\right.$ at the crash, inversion radius $\left.(r / a)_{q=1} \approx 0.08\right)$.

Nickel was injected as a trace by a laser blow-off system [56] during the ECRH phase. The impurity behavior was observed with a vacuum ultraviolet spectrometer consisting of a single line of sight in the plasma mid-plane. It measures the time evolution of the Ni XVII line at $24.92 \mathrm{~nm}$, whose time evolution is used as the Ni source term in the transport simulations. Two soft-x-ray cameras with a $2 \mathrm{~ms}$ time resolution provide information on impurity radiation in the plasma core. Details on these diagnostics can be found in [56, 57].

The radial transport analysis of Nickel is carried out using the ITC code [56]. It solves the system of continuity equations for all the ionization stages of the injected impurity. The impurity flux for each ionization stage results from diffusion and convection:

$$
\vec{\Gamma}_{z}=-D_{z}(r) \vec{\nabla} n_{z}(r)+\vec{V}_{z}(r) n_{z}(r)
$$

where $D_{z}(r)$ is the diffusion coefficient and $\vec{V}_{z}(r)$ the convection velocity. We assume that the diffusion coefficient and the convection velocity are independent of the ionization stage. 
Tore Supra
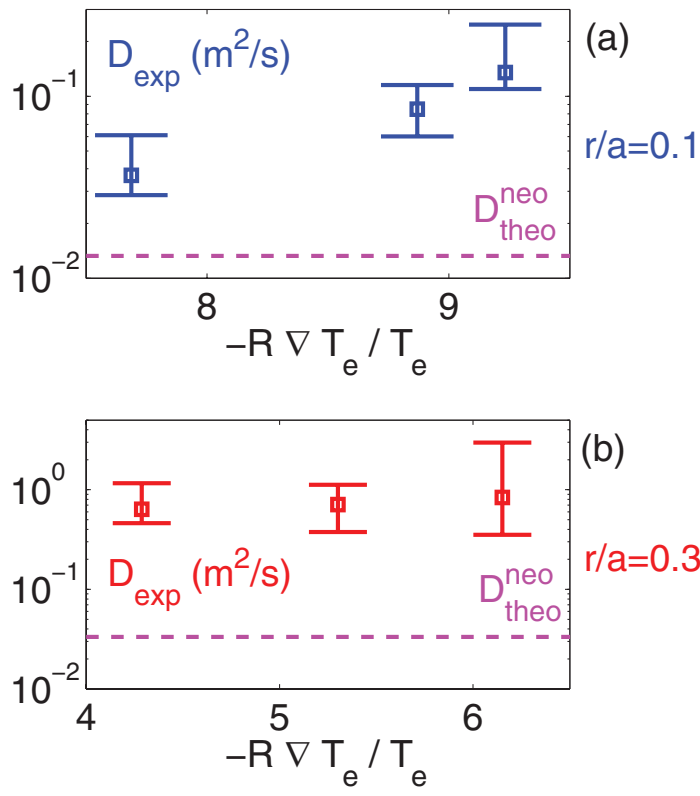

Figure 8. Experimental diffusion coefficient of nickel as a function of the normalized logarithmic electron temperature gradient at $r / a=0.1(a)$ and $r / a=0.3(b)$ in Tore Supra. The neoclassical diffusion coefficient is shown for both cases by a dotted line.

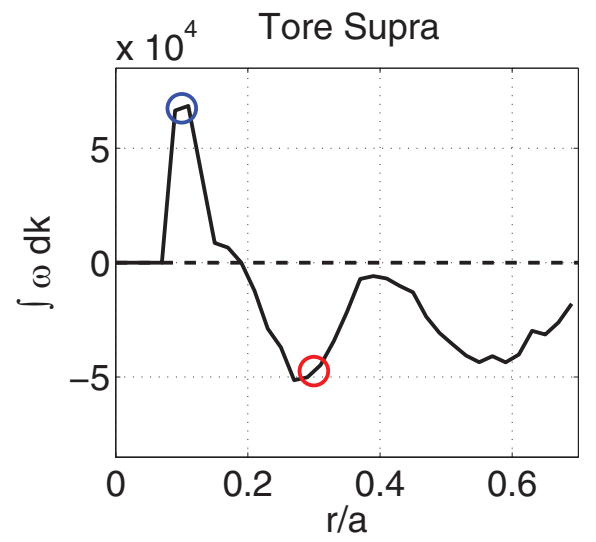

Figure 9. Radial profile of the integral over the wavenumber $\mathrm{k}$ of the spectrum of the most unstable mode frequency (Tore Supra \#40801). Circles correspond to the radii at which the diffusion coefficient in shown on figure 8 .

Starting from an initial guess, ITC solves the system of continuity equations. It then reconstructs the VUV, bolometric and soft x-ray brightnesses. With the help of a genetic algorithm, the code converges towards the transport coefficient profiles best matching the measurements [55].

The analysis performed for the three different ECRH deposition schemes shows that the diffusion coefficient at $r / a=0.1$ depends strongly on the normalized temperature gradient $R / L_{T_{e}}$ (figure $\left.8(a)\right)$. On the contrary, at $r / a=0.3$, the diffusion coefficient is almost independent of $R / L_{T_{e}}$ (figure $8(b)$ ).

\subsection{Comparison with linear gyro-kinetic simulations}

This difference is interpreted in the frame of the theory of turbulent transport. The existence of two regions [55] are

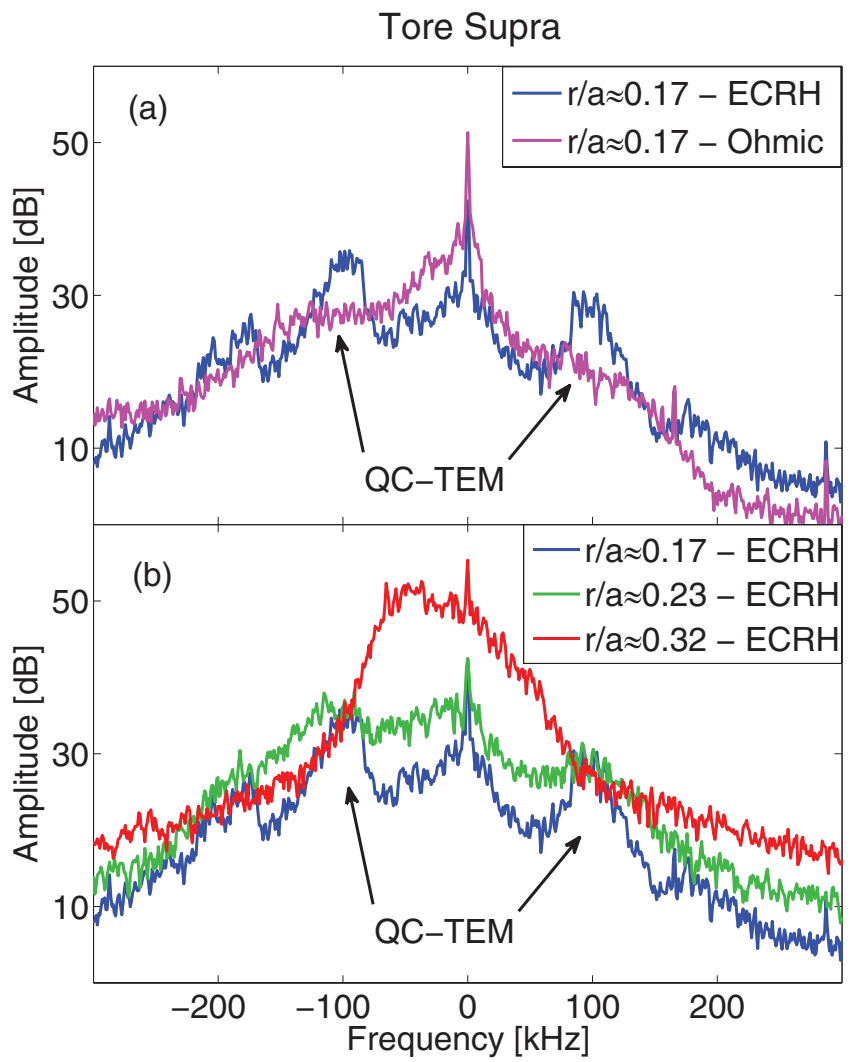

Figure 10. Fluctuation spectra of \#40801 from Tore Supra reflectometry. (a) shows the difference between ECRH and Ohmic phase and $(b)$ shows three different radial position during the ECRH phase. QC-TEM are clearly destabilized only below $r / a \approx 0.2$ in the ECRH phase.

observed in linear gyrokinetic simulations (see figure 9) performed with the QuaLiKiz code [4]. In the core, turbulence is dominated by electron modes $(r / a<0.2)$. With TEM dominated turbulence, the transport characteristics are affected by the electron temperature hence explaining figure $8(a)$. Further out, $(r / a>0.2)$, ITG modes are found dominant. The fact that ITG modes are independent of the electron temperature gradient is consistent with diffusion coefficients independent on $R / L_{T_{e}}$ (see figure $8(b)$ ).

\subsection{Fluctuation measurements: observation of QC-TEM in reflectometry spectra}

In the ECRH phase, as we could expect for TEM, the QC-TEM amplitude is enhanced compared to the Ohmic phase (see figure 10(a)) as previously reported in T-10 [58]. This is shown in figure $10(b)$ for the inner ECRH deposition ( $r / a=0.35$ for both gyrotrons) but it has been observed for the three configurations of ECRH power deposition.

In addition, a radial scan performed by changing the reflectometer frequency were done in the same discharge. It shows that QC-TEM are large in the core $(r / a<0.2)$, damped between $0.2<r / a<0.3$ and disappear further out $(r / a>0.3)$. These observations of QC-TEM correspond to the expectation for TEM deduced from the Nickel transport analysis and the linear gyro-kinetic simulation. 
It is not possible to conclude on the amplitude of QC-TEM during the scan of $R / L_{T_{e}}$ because the reflectometer settings were not similar, therefore the noiselevel and the radial position slightly change between the three discharges. However we can notice that the QC-TEM energy as defined in section 4.1.1 remains high in the three ECRH cases for $r / a<0.2$. It is estimated to be between 1.73 and $1.88 \mathrm{~dB}$ $\mathrm{kHz}^{-1}$, which corresponds to the high range of the values observed in the LOC regime shown in the previous section (see figure 5).

Thus both in terms of amplitude and radial location, observations of QC-TEM coincide with conditions where TEM are expected to be unstable: in the ECRH phase, strong QC-TEM are detected in the core $(r / a<0.2)$ where TEM are expected to be dominant. At $r / a \approx 0.3$ where QC-TEM are not observed, both the growth rate computation and the experimental Nickel diffusion coefficient indicate that turbulence is driven by ITG.

\section{Conclusion and perspectives}

QC-TEM with a poloidally ballooned amplitude have been observed from the core to the gradient region at $k_{\perp} \rho_{i} \leqslant 1$. They have been linked to TEM instabilities by two different methods in three tokamaks of distinct shapes and sizes (Tore Supra, TEXTOR and JET). ECRH increases QC-TEM amplitude only in the core region of Tore Supra where Nickel transport and linear growth rate computation support a TEMdominated turbulence. In Ohmic plasmas of JET, TEXTOR and Tore Supra, QC-TEM disappear during density ramp-up and current ramp-down. In Tore Supra and TEXTOR, their disappearance has been determined to occur at the LOCSOC transition.

Detection of QC-TEM can now be used to study the role of TEM in transport. However, the scope of this type of analysis has to be assessed since QC-modes may not systematically appear in regions where turbulence is dominated by TEM. Differences between ITG and TEM spectra must also be theoretically investigated to understand the reason why QC oscillations can show-up only when TEM are driven unstable in the plasma core region and whether there is a link with the QC modes observed at the plasma edge. To answer these open issues, an ongoing work is dedicated to the comparison of frequency fluctuation spectra from nonlinear gyrokinetic simulations and reflectometry measurements in the LOC and the SOC regimes [59-61].

\section{Acknowledgments}

The authors thank D. Villegas, J. Bernardo and E. Joffrin for their valuable help. This work, in partnership with ATEM, is financially supported by the 'Conseil regional ProvenceAlpes-Côte d'Azur'. It has been carried out within the framework of the Erasmus Mundus International Doctoral College in Fusion Science and Engineering (FUSION-DC) and the EUROfusion Consortium. It has received funding from the Euratom research and training programme 2014-2018 under grant agreement No 633053. The views and opinions expressed herein do not necessarily reflect those of the European Commission.

\section{References}

[1] Garbet X. et al 2004 Physics of transport in tokamaks Plasma Phys. Control. Fusion 46 B557

[2] Malinov P. and Zonca F. 2005 Ion temperature gradient modes and the fraction of trapped electrons J. Plasma Phys. 71301

[3] Conway G.D., Angioni C., Dux R., Ryter F., Peeters A.G., Schirmer J., Troester C., CFN Reflectometry Group and the ASDEX Upgrade team 2006 Observations on core turbulence transitions in asdex upgrade using doppler reflectometry Nucl. Fusion $\mathbf{4 6}$ S799

[4] Bourdelle C., Garbet X., Imbeaux F., Casati A., Dubuit N., Guirlet R. and Parisot T. 2007 A new gyrokinetic quasilinear transport model applied to particle transport in tokamak plasmas Phys. Plasmas $\mathbf{1 4} 112501$

[5] Arnichand H. et al 2014 Quasi-coherent modes and electrondriven turbulence Nucl. Fusion 54123017

[6] Krämer-Flecken A., Dreval V., Soldatov S., Rogister A., Vershkov V. and the TEXTOR-team 2004 Turbulence studies with means of reflectometry at textor Nucl. Fusion $\mathbf{4 4} 1143$

[7] Vershkov V.A., Shelukhin D.A., Soldatov S.V., Urazbaev A.O., Grashin S.A., Eliseev L.G., Melnikov A.V. and the T-10 team 2005 Summary of experimental core turbulence characteristics in Ohmic and electron cyclotron resonance heated discharges in T-10 tokamak plasmas Nucl. Fusion 45 S203

[8] Krämer-Flecken A., Soldatov S., Xu Y., Arnichand H., Hacquin S., Sabot R. and the TEXTOR-team 2015 Long range correlation properties of quasi coherent modes at textor accepted in New J. Phys. 17073007

[9] White A.E. et al 2010 Simultaneous measurement of core electron temperature and density fluctuations during electron cyclotron heating on diii-d Phys. Plasma 17020701

[10] White A.E. et al 2010 Measurements of the cross-phase angle between density and electron temperature fluctuations and comparison with gyrokinetic simulations Phys. Plasmas 17056103

[11] Hillesheim J.C. et al 2013 Observation of a critical gradient threshold for electron temperature fluctuations in the DIII-D tokamak Phys. Rev. Lett. 110045003

[12] Hillesheim J.C. et al 2013 Experimental characterization of multiscale and multifield turbulence as a critical gradient threshold is surpassed in the DIII-D tokamak Phys. Plasmas 20056115

[13] Ernst D.R. et al 2014 Controlling h-mode particle transport with modulated electron heating in DIII-D and alcator c-mod via tem turbulence Proc. 25th IAEA Conf. on Fusion Energy (Saint Petersburg, Russia) EX/2-3

[14] Rice J.E. et al 2011 Rotation reversal bifurcation and energy confinement saturation in tokamak Ohmic 1-mode plasmas Phys. Rev. Lett. 107265001

[15] Rice J.E. et al 2011 Observations of core toroidal rotation reversals in alcator c-mod Ohmic l-mode plasmas Nucl. Fusion $\mathbf{5 1} 083005$

[16] Rice J.E. et al 2012 Ohmic energy confinement saturation and core toroidal rotation reversal in alcator c-mod plasmas Phys. Plasmas 19056106

[17] Rice J.E. et al 2013 Non-local heat transport, rotation reversals and up/down impurity density asymmetries in alcator c-mod Ohmic 1-mode plasmas Nucl. Fusion 53033004

[18] Chapman B.E., the MST team and Collaborators 2014 Overview of results from the mst reversed field pinch experiment Proc. 25th IAEA Conf. on Fusion Energy (Saint Petersburg, Russia) OV/5-3 
[19] da Graça S., Conway G.D., Viezzer E., Silva A., Cupido L., Manso M.E. and ASDEX Upgrade Team 2013 Poloidal velocity of mhd modes at the edge of the asdex upgrade tokamak 40th EPS Conf. (Espoo, Finland)

[20] Diallo A. et al 2014 Observation of edge instability limiting the pedestal growth in tokamak plasmas Phys. Rev. Lett. 112115001

[21] Wang Q. et al 2014 New edge coherent mode providing continuous transport in long-pulse h-mode plasmas Phys. Rev. Lett. 112185004

[22] Zhong W. 2014 The role of edge plasma instabilities in dynamical evolution of pedestal in the hl-2a tokamak Proc. 25th IAEA Conf. on Fusion Energy (Saint Petersburg, Russia) EX/P7-23

[23] Greenwald M. et al 1999 Characterization of enhanced d highconfinement modes in alcator c-mod Phys. Plasmas 61943

[24] Snipes J.A., LaBombard B., Greenwald M., Hutchinson I.H., Irby J., Lin Y., Mazurenko A. and Porkolab M. 2001 The quasi-coherent signature of enhanced $\mathrm{d} h$-mode in alcator c-mod Plasma Phys. Control. Fusion 43 L23

[25] Terry J.L. et al 2005 Transport phenomena in the edge of alcator c-mod plasmas Nucl. Fusion $\mathbf{4 5} 1321$

[26] Golfinopoulos T. et al 2014 External excitation of a shortwavelength fluctuation in the alcator c-mod edge plasma and its relationship to the quasi-coherent mode Phys. Plasmas 21056111

[27] Terry J.L., Brunner D., Davis E., Greenwald M., Hughes J.W., LaBombard B., Golfinopoulos T. and Alcator C-Mod Team 2014 New insights on boundary plasma turbulence and the quasi-coherent mode in alcator c-mod using a mirror langmuir probe Phys. Plasmas 21056108

[28] Whyte D.G. et al 2010 I-mode: an h-mode energy confinement regime with 1-mode particle transport in alcator c-mod $\mathrm{Nucl}$. Fusion $\mathbf{5 0} 105005$

[29] White A.E., Phillips P., Whyte D.G., Hubbard A.E., Sung C., Hughes J.W., Dominguez A., Terry J. and Cziegler I. 2011 Electron temperature fluctuations associated with the weakly coherent mode in the edge of i-mode plasmas Nucl. Fusion $\mathbf{5 1} 113005$

[30] Cziegler I. et al 2013 Fluctuating zonal flows in the i-mode regime in alcator c-mod Phys. Plasmas 20055904

[31] Krämer-Flecken A., Soldatov S., Vowinkel B. and Mueller P. 2010 Correlation reflectometry at textor Rev. Sci. Instrum. 81113502

[32] Sabot R., Sirinelli A., Chareau J.-M. and Giaccalone J.-C. 2006 A dual source d-band reflectometer for density profile and fluctuations measurements in Tore-Supra Nucl. Fusion 46 S685

[33] Cupido L. and Meneses L. 2013 Recent hardware developments on synthetized sources for reflectometry hopping systems at IPFN 11th Int. Reflectometry Workshop (Palaiseau, France)

[34] Sirinelli A., Meneses L. and JET-EFDA Contributors 2013 Density fluctuation measurements of JET h-mode plasmas using a radial correlation reflectometer 11th Int. Reflectometry Workshop (Palaiseau, France)

[35] Conway G.D., Schirmer J., Klenge S., Suttrop W., Holzhauer E. and the ASDEX Upgrade Team 2004 Plasma rotation profile measurements using doppler reflectometry Plasma Phys. Control. Fusion 46951

[36] Conway G.D. 1999 Effects of reflectometer asymmetries on fluctuation measurements Plasma Phys. Control. Fusion 4165

[37] Krämer-Flecken A. 2013 Measurement of long range correlations at textor 11th Int. Reflectometry Workshop (Palaiseau, France)
[38] Gott Yu.V. 2007 On artsimovichs formula Fiz. Plazmy 33958

[39] Brower D.L., Peebles W.A., Kim S.K., Luhmann N.C., Tang W.M. and Phillips P.E. 1987 Observation of a highdensity ion mode in tokamak microturbulence Phys. Rev. Lett. 5948

[40] Rettig C.L., Rhodes T.L., Leboeuf J.N., Peebles W.A., Doyle E.J., Staebler G.M., Burrell K.H. and Moyer R.A. 2001 Search for the ion temperature gradient mode in a tokamak plasma and comparison with theoretical predictions Phys. Plasmas 82232

[41] Garbet X. et al 1992 Turbulence and energy confinement in Tore Supra Ohmic discharges Nucl. Fusion 322147

[42] Simmet E.E. and the ASDEX Team 1996 Statistical analysis of the global energy confinement time in Ohmic discharges in the asdex tokamak Plasma Phys. Control. Fusion 38689

[43] Angioni C., Peeters A.G., Ryter F., Jenko F., Conway G.D., Dannert T., Fahrbach H.U., Reich M., Suttrop W. and Fattorini L. 2005 Relationship between density peaking, particle thermodiffusion, Ohmic confinement, and microinstabilities in asdex upgrade 1-mode plasmas Phys. Plasmas 12040701

[44] Angioni C. et al 2011 Intrinsic toroidal rotation, density peaking and turbulence regimes in the core of tokamak plasmas Phys. Rev. Lett. 107215003

[45] Sung C. et al 2013 Changes in core electron temperature fluctuations across the Ohmic energy confinement transition in alcator c-mod plasmas Nucl. Fusion 53083010

[46] Gao C. et al 2014 Non-local heat transport in alcator c-mod Ohmic 1-mode plasmas Nucl. Fusion 54083025

[47] Shimomura Y. 1985 Empirical scaling of energy confinement time in 1-mode plasma and optimised mode and some consideration of reactor core plasma in tokamak (Japanese Atomic Energy Research Institute) Report JAERI-M-85-080

[48] Bernardo J. 2015 Density impact on toroidal rotation in Tore Supra: experimental observations and theoretical investigation Plasma Phys. Control. Fusion 57035002

[49] Bortolon A., Duval B.P., Pochelon A. and Scarabosio A. 2006 Observation of spontaneous toroidal rotation inversion in ohmically heated tokamak plasmas Phys. Rev. Lett. 97235003

[50] Duval B.P., Bortolon A., Karpushov A., Pitts R.A., Pochelon A., Sauter O., Scarabosio A., Turri G. and the TCV Team 2008 Spontaneous 1-mode plasma rotation scaling in the TCV tokamak Phys. Plasmas 15056173

[51] Hornung G. 2013 Study of plasma turbulence by ultrafast sweeping reflectometry on the Tore Supra tokamak $P h D$ Thesis Aix-Marseille Université

[52] Weynants R.R. et al 1999 Overview of radiative improved mode results on textor-94 Nucl. Fusion 391637

[53] Shi Y.J. et al 2013 Ech effects on toroidal rotation: kstar experiments, intrinsic torque modelling and gyrokinetic stability analyses Nucl. Fusion $\mathbf{5 3} 113031$

[54] Villegas D., Guirlet R., Bourdelle C., Hoang G.T., Garbet X. and Sabot R. 2010 Experimental electron temperature gradient dependence of heavy impurity transport in fusion devices Phys. Rev. Lett. 105035002

[55] Villegas D., Guirlet R., Bourdelle C., Garbet X., Hoang G.T., Sabot R., Imbeaux F. and Ségui J.L. 2014 Experimental and theoretical study of nickel transport dependence on gradients in Tore Supra Nucl. Fusion 54073011

[56] Parisot T., Guirlet R., Bourdelle C., Garbet X., Dubuit N., Imbeaux F. and Thomas P.R. 2008 Experimental impurity 
transport and theoretical interpretation in a Tore Supra lower-hybrid heated plasma Plasma Phys. Control. Fusion 50055010

[57] Guirlet R., Villegas D., Parisot T., Bourdelle C., Garbet X., Imbeaux F., Mazon D. and Pacella D. 2009 Anomalous transport of light and heavy impurities in Tore Supra Ohmic, weakly sawtoothing plasmas $\mathrm{Nucl}$. Fusion 49055007

[58] Vershkov V.A. et al 2013 Nucl. Fusion 53083014
[59] Citrin J., Arnichand H., Bourdelle C., Bernardo J., Garbet X. Hacquin S. and Sabot R. 2014 Progress in understanding quasi-coherent modes through gyrokinetic simulation Joined US-EU TTF Workshop (Culham, UK)

[60] Arnichand H. et al 2015 Identification of trapped electron modes in fluctuation spectra 42th European Physical Society Conf. on Plasma Physics (Lisbon, Portugal)

[61] Arnichand H. et al 2015 Plasma Phys. Control. Fusion submitted 